\title{
Grant-free Radio Access IoT Networks: Scalability Analysis in Coexistence Scenarios
}

\author{
Meysam Masoudi ${ }^{\dagger}$, Amin $_{\text {Azari }}^{\dagger}$, Emre Altug Yavuz ${ }^{\ddagger}$, and Cicek Cavdar ${ }^{\dagger}$ \\ $\dagger$ Communication Systems Department, KTH Royal Institue of Techonology, Sweden \\ $\ddagger$ Ericsson AB, Stockholm, Sweden \\ Email:\{masoudi,aazari,cavdar\}@kth.se,emre.yavuz@ericsson.com
}

\begin{abstract}
IoT networks with grant-free radio access, like SigFox and LoRa, offer low-cost durable communications over unlicensed band. These networks are becoming more and more popular due to the ever-increasing need for ultra durable, in terms of battery lifetime, IoT networks. Most studies evaluate the system performance assuming single radio access technology deployment. In this paper, we study the impact of coexisting competing radio access technologies on the system performance. Considering $\mathcal{K}$ technologies, defined by time and frequency activity factors, bandwidth, and power, which share a set of radio resources, we derive closed-form expressions for the successful transmission probability, expected battery lifetime, and experienced delay as a function of distance to the serving access point. Our analytical model, which is validated by simulation results, provides a tool to evaluate the coexistence scenarios and analyze how introduction of a new coexisting technology may degrade the system performance in terms of success probability and battery lifetime. We further investigate solutions in which this destructive effect could be compensated, e.g., by densifying the network to a certain extent and utilizing joint reception.
\end{abstract}

Index Terms-battery lifetime, IoT, LoRa, LPWA network, interference modelling.

\section{INTRODUCTION}

The number of physical objects being connected to the Internet is growing at an unprecedented rate, which is realizing the idea of the Internet of Things (IoT) or the Internet of everything. As one of the major drivers of 5G, it is important to provide (i) scalable (ii) low-cost and (iii) ultra-durable connectivity for the future IoT networks [1]. Supporting the connectivity over future cellular networks has been a main study item in 3GPP, and several revolutionary and evolutionary connectivity solutions have been proposed/standardized [1]. However, the existing solutions do not consider jointly the scalability, durability, and cost.

The IoT devices are supposed to send short payload-size packets sporadically [2]. As the number of connected devices increases excessively, the signal and control overhead becomes a burden for the conventional grant-based radio access protocol commonly used in cellular networks due to the excessive control signaling [3]. As a promising solution, grant-free radio access has attracted a great deal of attention in recent years. In grant-free access, once a packet is triggered at the device, it is transmitted without any handshaking or authentication process. Several existing IoT technologies benefit from a

This study is supported by EU Celtic Plus Project SooGREEN Service Oriented Optimization of Green Mobile Networks. grant-free radio access for providing low-cost long-battery lifetime connectivity, including SigFox and LoRa [4]. The common characteristic of these IoT technologies that distinguishes them from existing WiFi and cellular solutions, used for short-range high-bandwidth connectivity and long-range mobile connectivity respectively, are leveraging narrowband communication to cover a large area with minimum possible power consumption at the devices. The low-power wide-area (LPWA) networks are expected to share 60 percent of the IoT market among themselves, a number that is expected to grow over time, and hence the competition between LPWA technologies is becoming intense [5]. Regarding the increasing number of IoT technologies aiming at providing large-scale IoT connectivity by reusing a set of radio resources, it is of paramount importance to investigate the mutual impacts of coexisting technologies on each other.

\section{A. Literature Study}

In [5] and [6], major IoT solutions over licensed and unlicensed bands have been introduced, and their challenges in providing massive IoT connectivity have been figured out. A thorough battery lifetime analysis for unlicensed band solutions, including IEEE 802.15.4, BLE, SigFox and LoRa, has been presented in [7] based on the physical layer characteristics, i.e. operation protocols, connectivity states, and consumed energy in each state. Among solutions over unlicensed spectrum, SigFox and LoRa, as introduced in [5], are dominant solutions. Performance limits of LoRa have been investigated in [8]-[11]. In [8], scalability of single-gateway LoRa network has been investigated, and it has been shown that as the number of end-nodes increases, the impact on co-spreading factor increases, and hence, network becomes interference limited. Experimental results on the impact of interference from other LoRa nodes have been presented in [10]. In [11], performance limits of LoRa have been discussed, and it has been shown that besides the aforementioned limits, regulations govern the ISM band, e.g., duty cycle of operation, also limit the scalability of LoRa networks. The authors of [12], [13] experimentally evaluated the impact of potential interfering technologies reside in the ISM band. Their results illustrate a significant impact of interference from IoT devices already installed in smart homes, business parks, and etc., on the performance of LoRa and SigFox communications. In [14], the authors aim at bridging among solutions in the licensed 
and unlicensed band by presenting a grant-free access scheme over licensed spectrum for long battery lifetime demanding devices.

The literature study reveals that most previous studies have been focusing on the investigation of performance limits for single technology scenarios, and hence, the impact of coexistent technologies with partial time/frequency overlapping has been neglected. While the literature study of interference management for cellular and WiFi networks is mature [15], there is a crucial need to investigate the interference impact for large-scale, heterogeneous, and short-packet communication, which is the focus of this work. The main contributions of this paper are as follows.

- Deriving the closed-form expressions for the probability of successful transmission, battery lifetime, and delay for a network in which $\mathcal{K}$ heterogeneous technologies, defined by their transmit powers, time, and frequency activity factors, are reusing radio resources. Investigating the performance impact of coexisting technologies.

- Realizing joint reception as a solution to compensate the degradation due to the interference from other interfering technologies. Analyzing performance enhancement of the network. Figuring out the limits on the performance improvement.

The remainder of this paper is organized as follows. In Section III the system model is presented. In Section III closed-form expressions for the key performance indicators (KPIs) are derived. The performance evaluation is presented in Section IV Concluding remarks are summarized in Section V.

\section{System MODEL}

Assume a network with a massive number of IoT devices from $\mathcal{K}$ different technologies which are randomly distributed according to a spatial Poisson point process (PPP). Inside each technology, the pattern of packet generation and shared spectrum usage across different devices may differ from one another. Then, we define $K$ classes of devices in the network, where $K \geq \mathcal{K}$. By a class, we mean devices with a common pattern of shared spectrum usage, where the pattern includes time-frequency pattern of transmitted packets, range of carrier frequency, transmit power, and rate of packet generation at devices. To collect data from these devices, access points (APs) are deployed in the interest area. The density of APs and devices for $i$ th class is denoted by $\lambda_{i, a}$ and $\lambda_{i, b}$ respectively. Denote the carrier frequency and time-frequency support of each packet from a class $i$ device, by $f_{i}$ and $\omega_{i} \times T_{i} \mathrm{~Hz} \times \mathrm{sec}$, in which $f_{i, \min } \leq f_{i} \leq f_{i, \max } \sqrt{1}$. Also, $\mathcal{T}_{i}$ denotes the average time between two consecutive packet transmissions of a class $i$ device. As in [14], [16], we assume that the transmitted energy is uniformly distributed over its time-frequency support, i.e., over a rectangle of size $\omega_{i} \times T_{i} \mathrm{~Hz} \times \sec$ for class $i$. The channel

\footnotetext{
${ }^{1}$ The region for carrier frequency captures two facts. First, most IoT devices have cheap oscillators, and hence, the carrier frequency of the transmitted signals drifts from lower and higher frequency $\left(f_{i, \min }, f_{i, \max }\right)$ [14]. Second, in some IoT technologies like SigFox, IoT devices randomly change the carrier frequency in consecutive transmissions to make the communication robust.
}

TABLE I: Frequently used symbols.

\begin{tabular}{ll}
\hline Symbol & Definition \\
\hline$i$ and $j$ & The class indexes \\
$G_{X}(x) \triangleq \operatorname{si}(X \leq x)$ & CDF of random variable $X$ \\
$\mathcal{G}_{X}(x)$ & PDF of random variable $X$ \\
$f_{i} \in\left\{f_{i, \mathrm{mn}}, f_{i, \mathrm{mx}}\right\}$ & Carrier frequency \\
$K$ & Number of classes of devices \\
$\lambda_{i, a}, \lambda_{i, b}$ & Density of APs and devices \\
$\xi_{i, j}$ & Time activity factor \\
$v_{i, j}$ & Frequency activity factor \\
$T_{i}$ & Transmission time for a packet \\
$1 / \mathcal{T}_{i}$ & Generation rate of packets \\
$\gamma, \gamma_{\mathrm{th}}$ & SINR, minimum required SINR \\
$\mathcal{P}_{\mathrm{sc}}$ & Probability of successful transmission \\
$w_{i}$ & Signal bandwidth \\
$\alpha, \sigma$ & Pathloss exponent, 2/ $\alpha$ \\
$\mathcal{L}_{\mathcal{I}_{j}}(s)$ & Laplace functional of interference \\
$\mathbb{E}_{(\cdot)}$ & $\left(\mathcal{I}_{j}\right)$ \\
$E_{j}$ & Expectation operator \\
$\mathcal{E}_{j, a}, \mathcal{E}_{j, b}$ & Battery capacity in Joules \\
$p_{m, j}^{\text {av }}$ & Energy consumption of device in 1 \\
$P_{j}$ & reporting period and AP in unit time \\
\hline
\end{tabular}

gain consists of pathloss with the pathloss exponent of $\alpha$, and Rayleigh fading. The required signal to interference and noise ratio (SINR) threshold for successful signal decoding at an AP is denoted by $\gamma_{\mathrm{th}}$.

Problem Description and KPIs: The main goal of this paper is modeling the received interference from co-existing technologies, evaluating the system performance in presence of such interference, and finding solutions to compensate for the performance degradation due to the interference. The main KPIs of interest in this work are as follows.

1) Battery lifetime: Battery lifetime measures the time span between deployment of a device and when the device has its battery drained. Regarding the fact that most of IoT devices are battery driven, long battery lifetime is of great importance in most IoT applications. If batteries of IoT devices need to be replaced frequently, the human intervention, and hence maintenance cost, will be high, which limits the scalability of IoT networks.

2) Experienced delay: The experienced delay is defined as a delay from having packet ready at the device to successful reception of data at the access point. Characterizing the statistics of the experienced delay is important for IoT applications with stringent delay requirement.

\section{THEORETICAL ANALYSIS}

\section{A. Analytical Modeling of Transmission Success Probability}

In order to derive the success probability, we first analyze the case in which devices and APs have been paired, i.e., the transmitted packets from each device are received just by one 


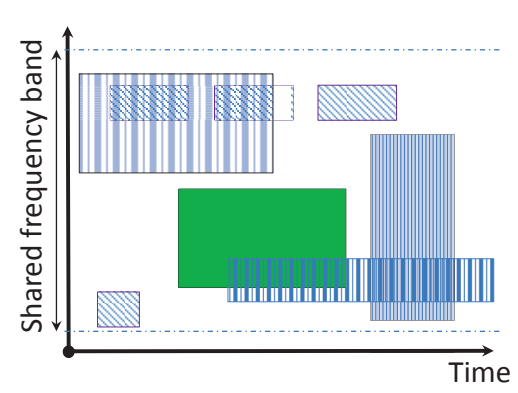

(a) (b)

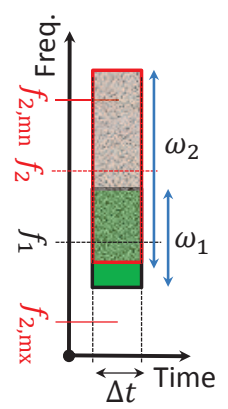

Fig. 1: Grant-free communications: (a) partial time-frequency overlapping of packets, and (b) average received power from an interfering device.

AP. Then in Section $\amalg$-D we extend our analysis to the multiAP scenario, in which several received copies of a packet in different APs are combined for performance enhancement. Let us first assume that each class $j$ device has been paired with only one class $j \mathrm{AP}$ which is always available to receive data. The duty cycle of class $i$ devices relative to class $j$ devices is denoted by $\mathcal{Z}(i, j) T_{i} / \mathcal{T}_{i}$. Where $\mathcal{Z}(i, j)=1$ when class $i$ and class $j$ refer to two different technologie: 2 , e.g. SigFox and LoRa, and $\mathcal{Z}(i, j)$ equals to the inverse of number of available orthogonal channels and codes in the technology otherwise? Then, the transmission success probability, $\mathcal{P}_{\text {sc }}$, for a class $j$ device at distance $d$ from the serving AP, is defined as:

$$
\mathcal{P}_{\mathrm{sc}}\left(j, d, \gamma_{\mathrm{th}}\right) \triangleq \operatorname{pr}\left(\gamma \geq \gamma_{\mathrm{th}}\right)
$$

where $\gamma$ is signal to noise and interference ratio, and is calculated as $\gamma=\mathcal{S} /[\mathcal{I}+\mathcal{N}]$, in which $\mathcal{S}, \mathcal{I}$, and $\mathcal{N}$ account for signal, aggregated interference, and noise powers. We start by the following proposition.

Proposition 3.1: The success probability for a packet transmitted by a device from class $j$ operating on carrier frequency $f_{j}$ at distance $d$ from the AP, is:

$$
\begin{gathered}
\mathcal{P}_{\mathrm{sc}}\left(j, d, \gamma_{\mathrm{th}}, f_{j}\right)=\exp \left(-\gamma_{\mathrm{th}} d^{\alpha} \mathcal{N} / P_{j}\right) \times \\
\prod_{i=1}^{K} \exp \left(-\xi_{i, j} \lambda_{i, b} \pi\left[\gamma_{\mathrm{th}} v_{i, j} P_{i} / P_{j}\right]^{\sigma} \mathbb{E}\left(h^{\sigma}\right) \Gamma(1-\sigma) d^{2}\right),
\end{gathered}
$$

in which $\sigma=2 / \alpha, \xi_{i, j}=\mathcal{Z}(i, j) T_{i} / \mathcal{T}_{i}$ is the time activity factor of class $i$ devices, $\mathbb{E}$ stands for expectation, $P_{i}$ is the transmit power of each class $i$ device, $v_{i, j}=$ $\mathcal{F}\left(f_{i, \mathrm{mn}}, f_{i, \mathrm{mx}}, \omega_{i}, \omega_{j}, f_{j}\right)$ accounts for the expected overlap between packets from $i$ and $j$ classes, and will be defined in Proposition 3.2

Proof: Starting from the definition of success probability,

\footnotetext{
${ }^{2}$ Note: two classes may refer to the same technology, e.g. two SigFox networks with different message generation rates, or two different technologies, e.g. SigFox and LoRa.

${ }^{3}$ For example in LoRa, we usually have 3 orthogonal channels and 7 spreading factor to select for sending data [5].
}

we have

$$
\begin{aligned}
\mathcal{P}_{\mathrm{sc}}\left(j, d, \gamma_{\mathrm{th}}, f_{j}\right) & =\operatorname{pr}\left(\gamma \geq \gamma_{\mathrm{th}}\right)=\operatorname{pr}\left(\mathcal{S}>\gamma_{\mathrm{th}}\left(\mathcal{N}+\mathcal{I}_{j}\right)\right) \\
& \stackrel{(a)}{=} \exp \left(-\gamma_{\mathrm{th}} d^{\alpha} \mathcal{N} / P_{j}\right) \mathbb{E}_{\mathcal{I}_{j}}\left(e^{-\gamma_{\mathrm{th}} d^{\alpha} \mathcal{I}_{j} / P_{j}}\right) \\
& =\left.\exp \left(-\gamma_{\mathrm{th}} d^{\alpha} \mathcal{N} / P_{j}\right) \mathcal{L}_{\mathcal{I}_{j}}(s)\right|_{s=\gamma_{\mathrm{th}} d^{\alpha} / P_{j}}
\end{aligned}
$$

where $\mathcal{I}_{j}$ represents the average aggregated received interference on the tagged packet from a class $j$ device and (a) is due to the independence of noise and interference, the first and the second terms in 3 , respectively. $\mathcal{L}_{\mathcal{I}_{j}}(s)$ is the Laplace functional of interference [17]. As $\mathcal{I}_{j}$ includes potential interference from all $K$ classes, we have

$$
\mathcal{I}_{j}=\sum_{i=1}^{K} \mathcal{I}_{i, j}, \quad \mathcal{I}_{i, j}=\sum_{x \in \Phi_{i}} h \ell(x) v_{i, j} P_{i} .
$$

In this expression, $\Phi_{i}$ is the set containing the locations of interfering class $j$ devices, $h$ is fading, $v_{i, j}$ represents the length of the overlapped frequency band between packets from class $i$ and class $j$ devices, and $\ell(x)$ is pathloss component. $\mathcal{L}_{\mathcal{I}_{i, j}}(s)$ is defined as [17]:

$$
\begin{aligned}
\mathcal{L}_{\mathcal{I}_{i, j}}(s) & \triangleq \mathbb{E}\left(\exp \left(-s \mathcal{I}_{i, j}\right)\right) \\
& =\mathbb{E}\left(\prod_{x \in \Phi_{i}} \exp \left(-s v_{i, j} P_{i} h r^{-\alpha}\right)\right),
\end{aligned}
$$

where the expectation is taken over both $\Phi_{i}$ and $h$. Also, in this expression $\ell(x)=r^{-\alpha}$ is assumed, where $r$ is the distance between interfering class $i$ device located at $x \in \Phi$, and the origin. Since fading is independent of the point process, the expectation operator can be moved inside the product, then we have:

$$
\begin{aligned}
\mathcal{L}_{\mathcal{I}_{i, j}}(s) & =\exp \left(-\int_{0}^{\infty} \mathbb{E}\left(\left[1-\exp \left(-s v_{i, j} P_{i} h r^{-\alpha}\right)\right]\right) \xi_{i, j} \lambda_{i, b} 2 \pi r d r\right), \\
& =\exp \left(-\xi_{i, j} \lambda_{i, b} \pi \mathbb{E}\left(h^{\sigma}\right) \Gamma(1-\sigma)\left[s v_{i, j} P_{i}\right]^{\sigma}\right) .
\end{aligned}
$$

Using (5) and (7), one can derive $\mathcal{L}_{\mathcal{I}_{j}}(s)$ as:

$\mathcal{L}_{\mathcal{I}_{j}}(s)=\prod_{i=1}^{K} \exp \left(-\xi_{i, j} \lambda_{i, b} \pi \mathbb{E}\left(h^{\sigma}\right) \Gamma(1-\sigma)\left[s v_{i, j} P_{i}\right]^{\sigma}\right)$.

By combining (4) and (8), the success probability is derived as presented in 2 .

For Rayleigh distributed fading, the success probability in (2) is rewritten as:

$$
\begin{aligned}
& \mathcal{P}_{\mathrm{sc}}\left(j, d, \gamma_{\mathrm{th}}, f_{j}\right)=\exp \left(-\gamma_{\mathrm{th}} d^{\alpha} \mathcal{N} / P_{j}\right) \\
& \prod_{i=1}^{K} \exp \left(-\xi_{i, j} \lambda_{i, b} \pi\left[\gamma_{\mathrm{th}} v_{i, j} P_{i} / P_{j}\right]^{\sigma} d^{2} / \operatorname{sinc}(\sigma)\right) .
\end{aligned}
$$

Proposition 3.2: Assume two devices transmit data simultaneously, as depicted in Fig. 1 b, with transmit powers of $P_{1}$ and $P_{2}$, and carrier frequencies of $f_{1}$ and $f_{2}$, where $f_{2}$ is a random variable in $\left[f_{2, \mathrm{mn}}, f_{2, \mathrm{mx}}\right]$, and follows a general distribution with cumulative distribution function (CDF) of $G_{f_{2}}(x)$. Then, the ratio between the expected length of the overlapped frequency band and $\omega_{1}$ is:

$$
\begin{gathered}
\mathcal{F}\left(f_{2, \mathrm{mn}}, f_{2, \mathrm{mx}}, \omega_{2}, \omega_{1}, f_{1}\right)=\int_{0}^{\min \left\{\omega_{1}, \omega_{2}\right\}}\left[G_{f_{2}}\left(f_{1}+\left[\omega_{1}+\omega_{2}\right] / 2-x\right)\right. \\
\left.-G_{f_{2}}\left(f_{1}-\left[\omega_{1}+\omega_{2}\right] / 2+x\right)\right] / \omega_{1} d x
\end{gathered}
$$

Proof: The CDF of length of the overlapped frequency 
band between packets from device 1 and 2 is:

$$
\begin{gathered}
G_{v}(x)=\operatorname{pr}(v \leq x)=\operatorname{pr}\left(f_{2} \geq f_{1}+\left[\omega_{1}+\omega_{2}\right] / 2-x\right) \\
\quad+\operatorname{pr}\left(f_{2} \leq f_{1}-\left[\omega_{1}+\omega_{2}\right] / 2+x\right), \\
=1-G_{f_{2}}\left(f_{1}+\left[\omega_{1}+\omega_{2}\right] / 2-x\right) \\
\quad+G_{f_{2}}\left(f_{1}-\left[\omega_{1}+\omega_{2}\right] / 2+x\right) .
\end{gathered}
$$

Given $y$ as a random variable with $\mathrm{CDF}$ of $G_{Y}(y)$, mean of $y$ is derived as $\int\left[1-G_{Y}(y)\right] d y$ [18]. Using this fact, deriving (10) from (11) is straightforward.

When $f_{2}$ is uniformly distributed in $\left[f_{2, \mathrm{mn}}, f_{2, \mathrm{mx}}\right]$, and

$$
f_{2, \mathrm{mx}}>f_{1}+\left[\omega_{1}+\omega_{2}\right] / 2 \text { and } f_{2, \mathrm{mn}}<f_{1}-\left[\omega_{1}+\omega_{2}\right] / 2
$$

hold, the ratio between expected length of the overlapped frequency band and $\omega_{1}$ is

$$
\mathcal{F}\left(f_{2, h}, f_{2, \mathrm{mx}}, \omega_{2}, \omega_{1}, f_{1}\right)=\omega_{2} /\left(f_{2, \mathrm{mx}}-f_{2, \mathrm{mn}}\right) .
$$

Finally, the average success probability over $f_{j}$ is derived as:

$$
\mathcal{P}_{\mathrm{sc}}\left(j, d, \gamma_{\mathrm{th}}\right)=\int_{f_{j, \mathrm{mn}}}^{f_{j, \mathrm{mx}}} \mathcal{G}_{f_{j}}(x) \mathcal{P}\left(j, d, \gamma_{\mathrm{th}}, x\right) d x
$$

in which $\mathcal{G}_{f_{j}}(x)=\partial G_{f_{j}}(x) / \partial x$ denotes the probability distribution function (PDF) of $f_{j}$ over $\left[f_{j, \mathrm{mn}}, f_{j, \mathrm{mx}}\right]$.

\section{B. Analytical Modeling of KPIs}

First, we investigate the delay, i.e., the time span between packet generation at the device and successful packet reception at the AP. Regarding the fact that each packet is transmitted with success probability $\mathcal{P}_{\text {sc }}\left(j, \gamma_{\text {th }}\right)$, the average experienced delay for a successfully received packet from a class $j$ device is derived as:

$$
D_{j}=\sum_{n=1}^{N_{\mathrm{tx}}}\left[n T_{j}+[n-1] T_{w_{j}}\right] \mathcal{P}_{\mathrm{sc}}\left(j, \gamma_{\mathrm{th}}\right)\left[1-\mathcal{P}_{\mathrm{sc}}\left(j, \gamma_{\mathrm{th}}\right)\right]^{n-1}
$$

where $N_{\text {tx }}$ denotes the maximum number of transmissions for a packet, and $T_{w_{j}}$ denotes the average waiting time between two retransmissions for class $j$ devices.

Now, we investigate the battery lifetime, i.e., the time span between deployment of a class $j$ device with battery capacity $E_{j}$ until when it has its battery drained. Regarding the fact that the reporting period for a class $j$ device is $T_{j}$, the expected battery lifetime is derived as:

$$
L_{j}=E_{j} \mathcal{T}_{j} / \mathcal{E}_{j, b},
$$

where $\mathcal{E}_{j, b}$ represents the average energy consumption per reporting period. Also, $\mathcal{E}_{j, b}$ can be modeled as:

$\mathcal{E}_{j, b}=P_{c} T_{a_{j}}+\left[P_{c}+\eta P_{j}\right] T_{j} \bar{n}_{j}+\left[\bar{n}_{j}-1\right]\left[P_{c} T_{w}+P_{r} T_{\text {ack }}\right]+P_{r} T_{\text {ack }}$,

where the first term denotes energy consumption in data gathering/processing, the second term indicates the energy consumption in data transmission, the third term indicates energy consumption in listening for ACK and waiting for retransmission, and the fourth term indicates energy consumption in receiving acknowledgment. Also, $P_{c}$ is a constant energy consumption in circuits, $\eta$ is the inverse power amplifier efficiency, $P_{i}$ is the transmit power, $T_{a_{j}}$ is the active time for

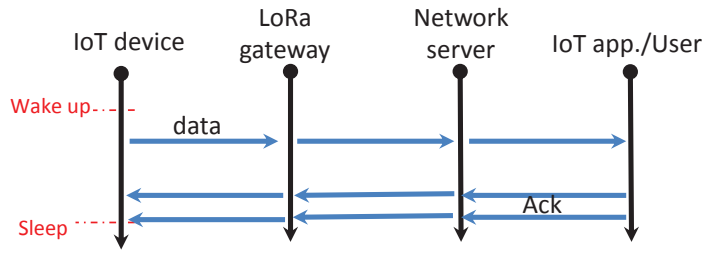

Fig. 2: Access protocol exchanges in LoRa

data gathering/processing, $T_{w}$ is the waiting time for receiving ack, $P_{r}$ is the energy consumption in receiving data, $T_{\mathrm{ack}}$ is the time-length of ack, and $\bar{n}_{j}$ denotes the average number of transmissions for a successful packet transfer, as follows:

$$
\bar{n}_{j}=\sum_{n=1}^{N_{\mathrm{tx}}} n \mathcal{P}_{\mathrm{sc}}\left(j, \gamma_{\mathrm{th}}\right) \mathcal{P}_{\mathrm{ack}}\left[1-\mathcal{P}_{\mathrm{sc}}\left(j, \gamma_{\mathrm{th}}\right) \mathcal{P}_{\mathrm{ack}}\right]^{n-1}
$$

where $\mathcal{P}_{\text {ack }}$ represents the probability of successful ACK reception. Considering neighboring APs as interfering nodes that their downlink transmissions can collide, $\mathcal{P}_{\text {ack }}$ can be found from 13 for given density, transmit power, and communication characteristics of the APs. Inserting $\mathcal{P}_{\text {sc }}$ from 13. into (16), we see how the increase in the number of coexisting devices operating in grant-free mode, decreases the success probability, increases the average number of retransmissions, and hence decreases the battery lifetime.

\section{Application to LoRa}

1) LoRa Technology: The LoRa wide area network provides seamless interoperability among IoT devices without any complex local installation requirements. LoRa is deployed in a star topology or cellular architecture in which a device is connected to a central network server via access points (APs) with the access protocol as depicted in Fig 2] The LoRa network manages spreading factors (SFs) for each device in order to optimize for the fastest possible data rate, which maximizes the network capacity. LoRa is utilizing chirp spread spectrum (CSS) as a modulation to maintain the immunity against the severe interference on unlicensed bandwidth and is fairly robust to multi-path fading and Doppler shifts [19]. The high resilience to the interferers is key to operate efficiently in the public ISM band. The main feature of CSS is that signals with different SFs can be distinguished and received simultaneously, even if they are transmitted at the same time on the same channel [20]. SFs, ranging from 7 to 12 , denote the number of chirps used to encode a bit. The higher chirp rate is, the better reconstruction of the received signal is attained, however, it stretches the transmission time [21]. For uplink transmission, the duty-cycle is 1\% in EU 868 [22], and devices use unslotted random access, similar to ALOHA. Downlink transmission can be done only during dedicated time intervals called receive windows, which follows successful uplink transmissions [23].

2) Analysis of LoRa: Assume LoRa devices and APs have been deployed in a 2-dimensional space, i.e., no intertechnology interference is considered. To get benefit from (13) for deriving the KPIs, we need to specify the time and frequency activity factors, i.e., $\xi_{i, j}$ and $v_{i, j}$ respectively. It is straightforward that $v_{i, j}$ is 1 , due to the intra-technology 
interference. Denoting the number of available channels in LoRa with $\mathcal{C}$, and the number of spreading factors (SF) available per channels as $|\mathrm{SF}|$, the time activity factor is modeled as:

$$
\xi_{i, j}=1 / \mathcal{C} \times 1 /|\mathrm{SF}| \times T_{i} / \mathcal{T}_{i},
$$

where $\mathcal{T}_{i}$ represents the average time between generation of two successive packets at a device, and $T_{i}$ represents the average transmission time for each packet. In LoRa protocol, devices wait for ACK within two windows after transmissions. If a node does not receive any ACK within the specified ACK windows, it transmits the packet again. ACKs in LoRa can be sent over a separate channel which is not used for transmission of devices' packets, and hence, the collision between data and ACK packets can be ignored. In this sense, the ACK success probability is as follows:

$$
\mathcal{P}_{\text {ack }}=\mathcal{P}_{\text {ack }, 1}+\mathcal{P}_{\text {ack }, 2}-\mathcal{P}_{\text {ack }, 1} \mathcal{P}_{\text {ack }, 2}
$$

where $\mathcal{P}_{\text {ack }, 1}$ and $\mathcal{P}_{\text {ack }, 2}$ are probabilities of receiving ACK in the first and second receiving windows, and can be derived as described in Section III-B Substituting 17) in (13), we can derive the success probability, i.e the probability of receiving packet at the AP successfully. Subsequently, one can insert the success probability in (14) and (16) in order to derive the delay expression.

\section{Joint Reception}

Since in grant-free access, there are no established connections between devices and APs, multiple APs may receive the signal from a device. Therefore, the received signals at the different APs can be utilized to improve the received SINR and consequently eliminate the destructive effect of interfere technologies. To do so, each AP sends the received signal to the IoT server where the received signals are combined using combining methods such as MRC.

1) Analysis of Joint Reception: Denote distance between a class $j$ device and $m$ th neighbor AP as $d_{m}$, where $m \in\{1, \cdots, \mathcal{M}\}$. Define event $e_{m}=\left\{\operatorname{SINR} \geq \gamma_{t h}\right\}$ at AP $m$, the event that the received SINR is greater than $\gamma_{t h}$ at AP $m$. We further extend the scenario to the case in which APs do not perform the decoding themselves but send the received signals to the network server for further processing and joint reception. In this case,

$$
\mathcal{P}_{s c}\left(j, \mathrm{~d}, \gamma_{\mathrm{th}}, f_{j}\right)=\operatorname{pr}\left(H(\Gamma, \Pi) \geq \gamma_{\mathrm{th}}\right)=1-G_{H(\Gamma, \Pi)}\left(\gamma_{\mathrm{th}}\right),
$$

in which $H(\Gamma, \Pi)$ is the function that describes the SINR gain achieved by combining, $G_{H(\Gamma, \Pi)}(x)=\operatorname{pr}(H(\Gamma, \Pi) \leq x)$ denotes $\mathrm{CDF}$ of $H(\Gamma, \Pi), p_{i, j}^{\mathrm{av}}$ denotes the fraction of time AP $i$ is in the listening mode, and

$$
\Gamma=\left[\gamma_{1, j}, \cdots, \gamma_{\mathcal{M}, j}\right] ; \Pi=\left[p_{1, j}^{\mathrm{av}}, \ldots, p_{\mathcal{M}, j}^{\mathrm{av}}\right] .
$$

An upper-bound on $H(\cdot)$ is achieved by maximum ratio combining (MRC), i.e.,

$$
H(\Gamma, \Pi)=\sum_{m=1}^{M} p_{m, j}^{\mathrm{av}} \gamma_{m, j}
$$

TABLE II: Simulation parameters.

\begin{tabular}{ll}
\hline Parameter & Value \\
\hline Signal bandwidth & $125 \mathrm{KHz}$ \\
Noise power density & $-174 \mathrm{dBm} / \mathrm{Hz}$ \\
Transmit power: RT, IT. & $20,14 \mathrm{dBm}$ \\
Frequency activity factor & $10^{-1}$ \\
Time activity factor & 0.01 \\
Density of devices & $10^{-2}$ devices $/ m^{2}$ \\
Battery capacity & $4000 \mathrm{joule}$ \\
$P_{c}, \gamma_{\text {th }}, \eta$ & $100 \mathrm{~mW}, 3 \mathrm{~dB}, 0.7$ \\
$T_{a}, T_{\text {ack }}$ & $2,1 \mathrm{sec}$ \\
$\mathcal{C},|\mathrm{SF}|$ & 3,7 \\
\hline
\end{tabular}

The CDF and PDF of $p_{m, j}^{\mathrm{av}} \gamma_{m, j}$ are derived as [18]:

$$
\begin{gathered}
G_{p_{m, j}^{\mathrm{av}} \gamma_{m, j}}(x)=1-\mathcal{P}_{\mathrm{sc}}\left(j, d_{m}, x / p_{m, j}^{\mathrm{av}}, f_{j}\right), \\
\mathcal{G}_{p_{m, j}^{\mathrm{av}} \gamma_{m, j}}(x)=\partial G_{p_{m, j}^{\mathrm{av}} \gamma_{m, j}}(x) / \partial x,
\end{gathered}
$$

in which $\mathcal{P}_{\text {sc }}$ has been found in $(9)$. Then, $G_{H(\Gamma, \Pi)}(x)$ is found as:

$$
G_{H(\Gamma, \Pi)}(x)=G_{p_{1, j}^{\mathrm{av}} \gamma_{1, j}} * \mathcal{G}_{p_{2, j}^{\mathrm{av}} \gamma_{2, j}} * \cdots * \mathcal{G}_{p_{\mathcal{M}, j}^{\mathrm{av}} \gamma_{\mathcal{M}, j}}(x),
$$

in which $*$ denotes the convolution. Now, the success probability is upperbounded as follows:

$$
\mathcal{P}_{\mathrm{sc}}\left(j, d, \gamma_{\mathrm{th}}\right)=\int_{f_{j, \mathrm{mn}}}^{f_{j, \mathrm{mx}}} \mathcal{G}_{f_{j}}(x)\left[1-\left.G_{H(\Gamma, \Pi)}\left(\gamma_{\mathrm{th}}\right)\right|_{f_{j}=x}\right] d x
$$

which can be evaluated given statistics of the interference, i.e., $\mathcal{G}_{f_{j}}(x)$. Now, by substituting (19) in (14) and (16), one can derive the delay and lifetime expressions.

\section{PERformance EVAluations}

In this section, we evaluate performance of a reference grant-free technology (RT), a simplified form of LoRa, while an interfering technology (IT) is active within the same frequency band. Assume we have APs and devices distributed over coverage area with PPP distribution. The transmit powers of IoT devices that belong to reference and competing technologies are set to 20 and $14 \mathrm{dBm}$. We assume that the reference technology has 3 channels in which devices can choose to send their packets. ACKs are sent over a separate channel so we do not have any collision between packets and ACKs. The pathloss exponent, $\alpha$, is 4 and we have Rayleigh fading with unit mean exponential distribution. In case of collision, each packet can be retransmitted 7 times. A packet transmission is successful if the SINR at the receiver is above the threshold, i.e., $\gamma_{t h}=3 \mathrm{dBm}$ (after taking spreading gain into account). For joint reception, we assume 3 nearest access points can receive the signal and send it to a central unit in which, the received signals are combined using maximum ratio combining scheme. Other simulation parameters can be found in Table III In Fig 3, the success probability as a function of distance to the serving access point has been plotted. One sees that adding coexisting technologies degrades the 


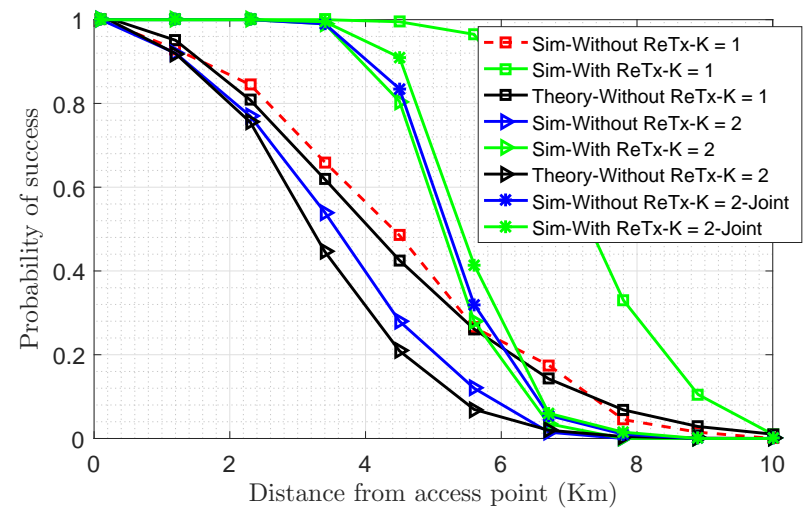

Fig. 3: Impact of joint reception and coexistent technologies

performance substantially. Also, we see how time diversity, i.e., retransmission, which is certainly achieved at the cost of a shorter device battery lifetime, increases the reliability of communications. We further observe that receiver diversity, i.e., joint reception of packets, which is achieved at the cost of more complex receivers, can significantly improve reliability of the system in presence of coexisting technologies reusing the same band. Also, one observes that there exist a point at which we cannot benefit from neither retransmissions (time diversity) nor joint reception (receiver diversity) to improve the performance, i.e., success probability. Thus, this is the point at which we need to change the communication protocol to make it more robust to the interference. In the case of LoRa, increasing the spreading factor brings more robust communications at the cost of longer transmission times, and hence, energy consumption per transaction. This observation pops out the idea to let each device to increase/decrease the spreading factor in use based on the received ACKs from the network. The average required number of transmissions for having the packet successfully received at the receiver has been demonstrated in Fig 4 One sees that having other active technologies on the same band increases the number of transmission retrials. One further observes that joint reception can well compensate the degradation caused by coexisting technologies, specially at points close to the access point where it outperforms the others due to the reinforced received signal strength. On the other hand, this reflects that by having both (i) denser networks, in which the average distance between a typical device and neighbor access points is not too large, and (ii) using joint reception, one can significantly improve the network performance.

In Fig 5, the expected battery lifetime of a typical device as a function of distance to the access point has been depicted. As we expect, by increasing the distance, more retransmissions are needed, which degrade the battery lifetime. Also, similar to what observed in Fig 4 joint reception can significantly prolong the battery lifetime specially in short distances, i.e., in dense AP deployment scenarios.

Fig 6 investigates the percentage of performance degradation in case of having multiple technologies active on the same band in comparison with the performance of single technology case. For devices close to the access point we do not see much

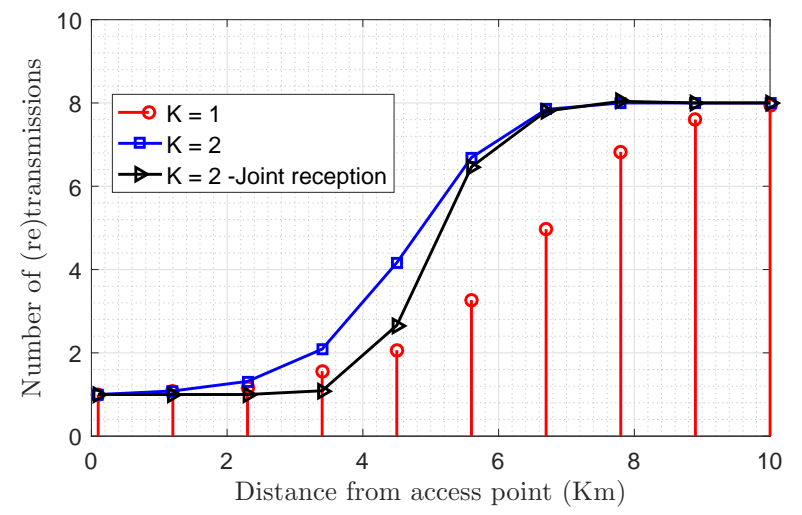

Fig. 4: Impact of joint reception on the number of (re)transmissions

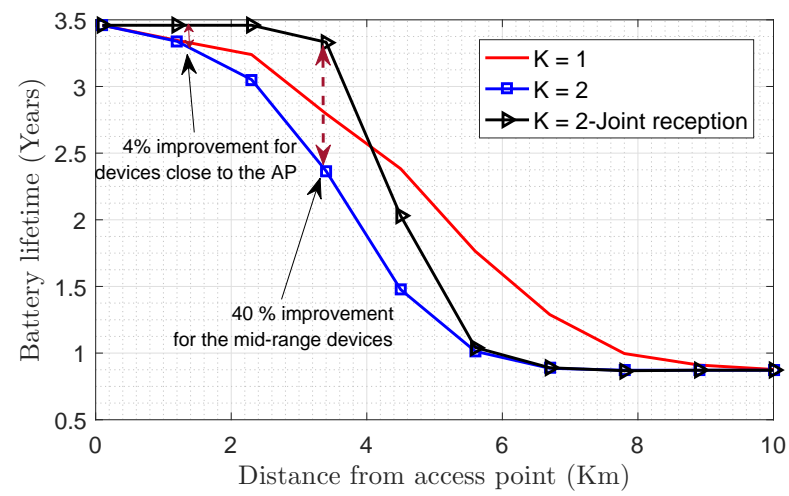

Fig. 5: Battery lifetime performance

performance degradation because the received signal strength is strong enough to decode the signal so the interference cannot affect the results heavily. For devices far away from the access point, the received signal is weak so that even in the absence of interferer the performance is very poor. The main impact of other technologies is observed for devices that are neither too close nor too far from the access point. In this region, the battery lifetime and success probability (for single transmission) are reduced by about $40 \%$ and 50 $\%$, respectively. But by joint reception, the destructive effect of interferes can be removed. In other words, reliability can be achieved by time diversity, i.e., increasing the number of retransmissions, as well as by receiver diversity, i.e., by joint reception, where the former is achieved at the cost of shorter battery lifetimes for devices and the latter is achieved at the cost of increase in CAPEX and OPEX of the network, i.e., deploying more access points and more advanced receivers at the network server.

\section{CONClusion}

In this paper, we have presented an analytical model to evaluate the performance of grant free IoT networks assuming that a radio spectrum is shared by competing radio access technologies. The model is developed with a cross layer approach which takes impacts of medium access control and physical layers into account. We have derived closed-form expressions to compute the successful transmission probability, battery 


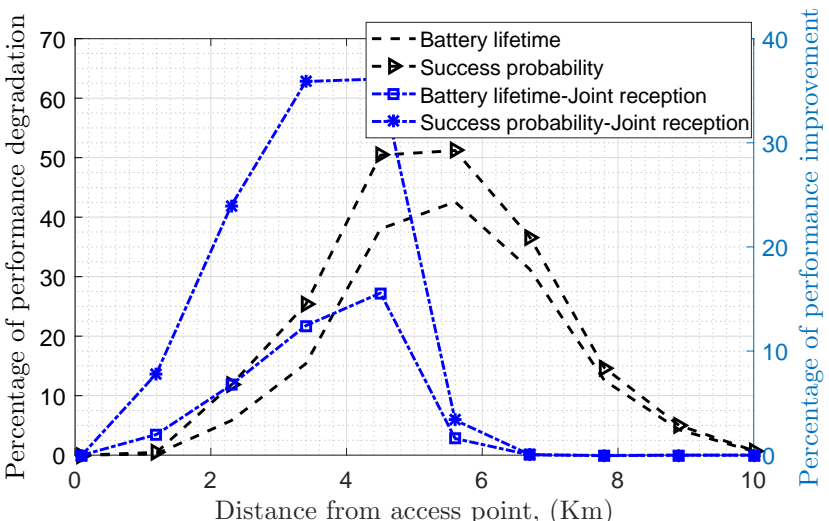

Fig. 6: Percentage of performance degradation

lifetime, and delay in the network. Analytical and simulation results indicated that existence of competing technologies could considerably degrade the performance. We have further demonstrated that using joint reception techniques, in which different access points receive the signal and relay it to the IoT server for joint reception, the performance degradation could be mitigated. Moreover, simulation results confirmed that there exists a distance from a typical access point, beyond where neither the number of retransmissions nor the number of cooperating APs contribute to successful reception of data. Characterizing this region is critical in system design, since to cover this region, one either needs to densify the network by deploying more APs, or increase the transmission power per data transfer at the device.

\section{REFERENCES}

[1] C. Mavromoustakis, G. Mastorakis, and J. M. Batalla, Internet of Things (IoT) in 5G mobile technologies. Springer, 2016, vol. 8.

[2] G. Miao, A. Azari, and T. Hwang, "E2MAC: Energy efficient medium access for massive M2M communications," IEEE Transactions on Communications, vol. 64, no. 11, pp. 4720-4735, 2016.

[3] Y. Du, B. Dong, Z. Chen, X. Wang, Z. Liu, P. Gao, and S. Li, "Efficient multi-user detection for uplink grant-free noma: Prior-information aided adaptive compressive sensing perspective," IEEE Journal on Selected Areas in Communications, 2017.

[4] É. Morin, M. Maman, R. Guizzetti, and A. Duda, "Comparison of the device lifetime in wireless networks for the internet of things," IEEE Access, vol. 5, pp. 7097-7114, 2017.

[5] W. Yang et al., "Narrowband wireless access for low-power massive internet of things: A bandwidth perspective," IEEE Wireless Communications, 2017.

[6] M. Centenaro, L. Vangelista, A. Zanella, and M. Zorzi, "Long-range communications in unlicensed bands: The rising stars in the IoT and smart city scenarios," IEEE Wireless Communications, vol. 23, no. 5, pp. 60-67, October 2016.

[7] É. Morin, M. Maman, R. Guizzetti, and A. Duda, "Comparison of the device lifetime in wireless networks for the internet of things," IEEE Access, vol. 5, pp. 7097-7114, 2017.

[8] O. Georgiou and U. Raza, "Low power wide area network analysis: Can LoRa scale?" IEEE Wireless Communications Letters, vol. 6, no. 2, pp. 162-165, 2017.

[9] N. Blenn and F. Kuipers, "LoRaWAN in the wild: Measurements from the things network," arXiv preprint arXiv:1706.03086, 2017.

[10] K. Mikhaylov, J. Petajajarvi, and J. Janhunen, "On LoRaWAN scalability: Empirical evaluation of susceptibility to inter-network interference," arXiv:1704.04257, 2017.

[11] F. Adelantado, X. Vilajosana, P. Tuset-Peiro, B. Martinez, and J. Melia, "Understanding the limits of LoRaWAN," arXiv:1607.08011, 2016.
[12] B. Vejlgaard et al., "Interference impact on coverage and capacity for low power wide area IoT networks," in Wireless Communications and Networking Conference (WCNC), 2017 IEEE. IEEE, 2017, pp. 1-6.

[13] M. Lauridsen, B. Vejlgaard, I. Z. Kovács, H. Nguyen, and P. Mogensen, "Interference measurements in the european $868 \mathrm{MHz}$ ISM band with focus on LoRa and SigFox," in Wireless Communications and Networking Conference (WCNC), 2017 IEEE. IEEE, 2017, pp. 1-6.

[14] A. Azari, P. Popovski, G. Miao, and C. Stefanovic, "Grant-Free Radio Access for Short-Packet Communications over 5G Networks," in Globecom 2017. IEEE, 2017, pp. 1-5.

[15] M. Masoudi, H. Zaefarani, A. Mohammadi, and C. Cavdar, "Energy efficient resource allocation in two-tier OFDMA networks with QoS guarantees," Wireless Networks, Jan 2017.

[16] Z. Li et al., "2D time-frequency interference modelling using stochastic geometry for performance evaluation in low-power wide-area networks," arXiv preprint arXiv:1606.04791, 2016.

[17] M. Haenggi, Stochastic geometry for wireless networks. Cambridge University Press, 2012.

[18] A. Papoulis, Probability and statistics. Prentice-Hall Englewood Cliffs, 1990, vol. 2.

[19] "Lora modulation basics," www.semtech.com Tech. Rep., 2015.

[20] D. Bankov, E. Khorov, and A. Lyakhov, "Mathematical model of LoRaWAN channel access," in Proceedings of the IEEE 18th International Symposium on A World of Wireless, Mobile and Multimedia Networks (WoWMoM), Macao, China, 2017, pp. 12-15.

[21] N. Blenn and F. Kuipers, "LoRaWAN in the wild: Measurements from the things network," arXiv preprint arXiv:1706.03086, 2017.

[22] F. Adelantado, X. Vilajosana, P. Tuset-Peiro, B. Martinez, and J. Melia, "Understanding the limits of LoRaWAN," arXiv preprint arXiv:1607.08011, 2016.

[23] D. Bankov, E. Khorov, and A. Lyakhov, "On the limits of LoRaWAN channel access," in Engineering and Telecommunication (EnT), 2016 International Conference on. IEEE, 2016, pp. 10-14. 